\title{
A Estratégia Saúde da Família para a Equidade de Acesso Dirigida à População em Situação de Rua em Grandes Centros Urbanos
}

The Family Health Strategy Focused on Access Equity and Targeted at the Homeless Population Living in Large Urban

\section{Centers}

\author{
Nivaldo Carneiro Junior \\ Médico Sanitarista. Doutor em Medicina Preventiva. Diretor do \\ Centro de Saúde Escola Barra Funda Dr. Alexandre Vranjac/Irman- \\ dade da Santa Casa de Misericórdia de São Paulo. Professor da \\ Faculdade de Ciências Médicas da Santa Casa de São Paulo. \\ Endereço: Rua Dr. Abraão Ribeiro, 283, CEP ol133-020, São Paulo, \\ SP, Brasil. \\ E-mail: csebf.dirtec®santacasasp.org.br

\section{Christiane Herold de Jesus} \\ Enfermeira Sanitarista. Mestre em Saúde Coletiva. Chefe de En- \\ fermagem do Centro de Saúde Escola Barra Funda Dr. Alexandre \\ Vranjac/Irmandade da Santa Casa de Misericórdia de São Paulo. \\ Endereço: Rua Dr. Abraão Ribeiro, 283, CEP ol133-020, São Paulo, \\ SP, Brasil. \\ E-mail: csebf.chenfœsantacasasp.org.br \\ Maria Angélica Crevelim \\ Enfermeira Sanitarista. Mestre em Administração em Enfermagem. \\ Secretária Executiva do Conselho de Ensino da Secretaria Municipal \\ de Saúde de São Paulo. \\ Endereço: Rua General Jardim, 36, CEP o1223-010, São Paulo, SP, \\ Brasil. \\ E- mail: acrevelimळprefeitura.sp.gov.br
}

\section{Resumo}

Este artigo relata experiência na implantação da atenção à saúde da população em situação de rua da cidade de São Paulo, visando à equidade de acesso às ações de saúde no Sistema Único de Saúde. As dificuldades de utilização dos serviços de saúde por esses indivíduos devem-se a: concepção do adoecimento-cuidado, organização técnico-administrativa dos serviços, preconceitos de profissionais e usuários e fragilidades de ações intersetoriais, entre outros. Ademais, observa-se prevalência maior de algumas condições na população de rua quando comparada às da população geral, como gestações seis vezes; alcoolismo 30 vezes e tuberculose 57 vezes - demonstrando, proporcionalmente, o grau de vulnerabilidade desse grupo. São necessários novos processos de trabalho pró-ativos na atenção à saúde que incorporem particularidades desses indivíduos, garantindo, assim, o acesso. Nesse sentido, a Estratégia Saúde da Família, por suas características, responde a tais necessidades. A partir de 2004, o projeto "A Gente na Rua" - fruto de parcerias entre a Secretaria Municipal de Saúde de São Paulo e instituições filantrópicas - foi implementado; inicialmente com o Programa de Agentes Comunitários de Saúde, e hoje com equipes de Saúde da Família. Conclui-se que, na cidade de São Paulo, a política adotada para a atenção à saúde da população de rua promoveu o acesso à atenção básica e processos de trabalho específicos. Questões como novas estratégias de cuidado aos que apresentam transtornos mentais, integralidade, longitudinalidade e intersetorialidade nas ações, entre outras aqui 
enunciadas, devem ser consideradas na constituição de futuras análises.

Palavras-chave: População em Situação de Rua; Estratégia de Saúde da Família; Equidade; Acesso; Atenção Primária à Saúde.
Abstract

This article reports the experience concerning the implementation of a health care program addressed to homeless people in the city of São Paulo, aiming at equity in the access to governmental health services. These individuals' difficulties in using the health services are due to their misconceptions about illness and care, health teams' and users' preconceptions, services' technical-administrative organization, and fragile intersector actions, among others. Additionally, this population is highly vulnerable to some health disorders, as shown by the high rates of alcoholism, tuberculosis and even pregnancy. Innovative actions as well as adequate health strategies addressing a real improvement in the access should be considered as essential. In this sense, the Family Health Strategy is a useful tool of the primary health care services. Since 2004, a Project named "A Gente na Rua”, which resulted from a partnership between the Municipal Health Department of São Paulo and philanthropic institutions, has been implemented, with the action of community health agent teams. It is concluded that, in the city of São Paulo, the adoption of this health policy targeted at the homeless has promoted better access to primary health care services. Moreover, it has created a more focused approach to this population. Despite the success of this strategy, we should take into account that remaining challenges have to be tackled in future projects, such as the need of new strategies focusing on homeless people with mental disorders, as well as the guarantee of quality care characterized by comprehensiveness, longitudinality and coordination, among others.

Keywords: Homeless Population; Family Health Strategy; Equity; Access; Primary Care. 


\section{Introdução}

A Estratégia de Saúde da Família (ESF), por suas possibilidades na mudança do perfil epidemiológico, transformou-se em política pública para o Sistema Único de Saúde (SUS), uma vez que “[...] introduziu uma visão ativa da intervenção em saúde, a de não 'esperar' a demanda 'chegar' para intervir, mas de agir sobre ela preventivamente, representando, assim, um instrumento real de reorganização da demanda” (Viana e Dal Póz, 1998, p. 10).

A organização do processo de trabalho para a garantia do acesso aos serviços de saúde nas comunidades assistidas pela ESF é uma das principais características que lhe confere singularidade e potência como modalidade tecnoassistencial em atenção básica.

Inserir-se no território, constituindo relações pró-ativas com a comunidade de sua responsabilidade, favorece o estabelecimento mais efetivo do cuidado em saúde, pois, além de permitir identificar problemas e priorizar intervenções, cria vínculos contínuos com os assistidos, possibilitando individualizar suas necessidades e organizar processos particulares de cuidado.

Este artigo pretende, portanto, descrever a experiência na implantação da ESF para atenção à saúde da população em situação de rua na cidade de São Paulo, apresentando, desse modo, não só a exposição de determinada política pública, mas também uma contribuição para o debate sobre a organização da equidade no acesso às ações de saúde no Sistema Único de Saúde (SUS).

\section{Equidade e Acesso aos Serviços de Saúde}

Alguns estudos tomam o acesso como categoria analítica para apreender as iniquidades da assistência à saúde de determinados grupos sociais, isto é, as desigualdades de acesso a recursos tecnológicos disponíveis para diferentes grupos sociais, que estabelecem a maior ou menor disponibilidade de cuidados à saúde (Giovanella e Fleury, 1996; Travassos e col., 2000).

Vários autores têm ressaltado o fato de que apenas ter serviços disponíveis não garante o acesso, uma vez que fatores relacionados ao consumo e à oferta influenciam sua utilização, como concentração de serviços em determinado nível de complexidade tecnológica, características culturais e econômicas da população, fluxos e rotinas dos serviços e graus de satisfação do usuário na utilização dos serviços, entre outros (Aday e Andersen, 1974; Penchansky e Thomas, 1981; Frenk, 1992; Wallace e Enriquez-Hass, 2001).

Para melhor compreender o acesso, os diversos aspectos de utilização dos serviços de saúde, que o influenciam de forma positiva ou negativa, podem ser agrupados nas seguintes dimensões: (1) disponibilidade - diz respeito à oferta de serviços; (2) acessibilidade - relaciona-se à proximidade e/ ou facilidade geográfica do serviço, como também às formas de trabalho organizadas para atenção, fluxos, horários, rotinas, prioridades técnicas, entre outros; e (3) aceitabilidade - dimensão que se relaciona com as características da população, isto é, suas crenças, condições socioeconômicas, graus de satisfação com o serviço utilizado, entre outros (Frenk, 1992; Aday, Andersen, 1974).

De acordo com Hortale e colaboradores (1999, p. 87), "a categoria acesso deve, portanto, ser vista não somente como geográfica e econômica, mas também organizacional e sociocultural. Ela pode descrever a capacidade que um serviço tem de dar cobertura a uma determinada população, ou o obstáculo a sua utilização, caracterizando uma interação que ocorre em um processo de produção de serviços”.

Nesse sentido, acesso constitui uma categoria importante para a análise dos sistemas de saúde, uma vez que revela dimensões de caráter social e político, necessariamente presentes nas formulações e implementações de políticas de saúde (Hortale e col., 2000).

Essa discussão é pertinente, sobretudo porque formas diferenciadas de acesso às políticas públicas por diversos segmentos sociais sempre orientaram as políticas adotadas pelo Estado brasileiro, chegando às décadas de 1970 e 1980 com padrões de profundas desigualdades sociais, o que decorre do próprio caráter excludente da sociedade. Ao prescrever que o Estado tem o dever de tornar disponível a saúde igualitária para todos os cidadãos, o Sistema Único de Saúde configura-se como uma política que 
pretende reverter esse quadro.

No processo de organização e implantação do SUS, a garantia de acesso aos serviços de saúde tem sido uma das grandes preocupações e um dos maiores desafios dos gestores. Nessa perspectiva, a ampliação e a efetiva ação da atenção básica em saúde tornam-se uma política prioritária do SUS, por se reconhecer que, nesse nível assistencial, é possível resolver os principais problemas de saúde, promover saúde e reorganizar o uso real dos demais níveis da atenção (Starfield, 2002). A Estratégia de Saúde da Família foi a modalidade tecnoassistencial eleita para dar conta dessa política.

\section{A Estratégia Saúde da Família}

O Programa Saúde da Família (PSF) no Brasil, formulado pelo Ministério da Saúde a partir de 1994, representa o ápice de várias propostas de mudanças - inspiradas pela reforma sanitária e pelos princípios do SUS - nos projetos de criação dos Distritos Sanitários e dos Sistemas Locais de Saúde (SILOS) (Silva Junior, 1998). O PSF emerge de um acúmulo de experiências desenvolvidas em algumas regiões do país, principalmente com o Programa de Agentes Comunitários de Saúde (PACS), criado em 1991, na esteira dos programas de agentes de saúde do Ceará e de Goiás (Crevelim, 2004).

A Saúde da Família é entendida como uma estratégia de reorientação do modelo assistencial, operacionalizada mediante a implantação de equipes multiprofissionais em unidades básicas de saúde. Essas equipes são responsáveis pelo acompanhamento de um número definido de famílias, localizadas em uma área geográfica delimitada, e atuam com ações de promoção da saúde, prevenção, recuperação, reabilitação de doenças e agravos mais frequentes e na manutenção da saúde dessa comunidade. Esse processo de acompanhamento estabelece, para as equipes de saúde da família, a necessidade de ultrapassar os limites classicamente definidos para a atenção básica no Brasil, especialmente no contexto do SUS (Brasil, 2009).

Como estratégia estruturante dos sistemas municipais de saúde, a Saúde da Família tem provocado um importante movimento no sentido de reordenar o modelo de atenção no SUS, já que busca maior racionalidade na utilização dos demais níveis assistenciais e produz resultados positivos nos principais indicadores de saúde das populações assistidas pelas equipes (Brasil, 2009).

No município de São Paulo, a Estratégia de Saúde da Família foi implantada pelo gestor municipal em 2001. Hoje conta com 850 equipes em atuação, envolvendo 4.915 agentes comunitários de saúde, que correspondem a $26,62 \%$ da população total da cidade (Brasil, 2009).

\section{A Estratégia de Saúde da Família para a População em Situação de Rua na Cidade de São Paulo}

A população em situação de rua tem presença marcante em regiões centrais das metrópoles, pois essas áreas parecem oferecer mais possibilidades de viabilizar seu "modo de andar a vida": grande concentração de serviços, baixa concentração de residências e alta circulação de pessoas que contribuem para a sua localização territorial.

A rua pode ora se constituir em abrigo para aqueles que, sem recursos, dormem circunstancialmente sob marquises de lojas, viadutos, bancos de jardim; ora ser, ainda, um modo de vida para os que já construíram nela seu hábitat e que estabelecem com ela uma complexa rede de relações (Vieira e col., 2004).

Trata-se de um grupo populacional heterogêneo, determinado, principalmente, pela relação variável entre o tempo na rua e os mais diversos vínculos sociais. Nessa perspectiva, faz-se interessante o agrupamento de três grandes situações que, de alguma maneira, ajudam a traçar o perfil desse grupo: ficar na rua (momento circunstancial), estar na rua (a rua como o local de pernoite) ou ser de rua (a rua torna-se um espaço de moradia de forma praticamente definitiva) (Vieira e col., 2004).

De acordo com a pesquisa realizada em 2003 (São Paulo, 2003), havia 10.49o pessoas vivendo em situação de rua na cidade de São Paulo, das quais 60 \% na região central. Atualmente, projeções não oficiais apontam número próximo de 13 mil.

Junto a essa população, o acesso aos serviços de saúde se apresenta, de modo geral, com significativos obstáculos para sua efetiva garantia, em especial 
na atenção primária, constituindo-se em um importante preditor de suas condições de saúde, lembrando que o padrão de risco observado é constantemente desvantajoso para indivíduos pertencentes a grupos sociais menos privilegiados. Aspectos relacionados às concepções do adoecimento e do processo do cuidado desses indivíduos também contribuem para sua não adesão. Todavia, questões referentes à própria organização do serviço exercem papel fundamental no acesso: exigência de documentação, restrição no atendimento da demanda espontânea, limites na atuação intersetorial, preconceitos, entre outras, criam vínculos precários (Carneiro Junior e col., 1998).

Frente ao quadro, são necessários novas abordagens e novos processos de trabalho na atenção à saúde dessa população, os quais introduzam em suas formulações a equidade no acesso aos serviços de saúde e que sejam orientados para o que se denomina "discriminação positiva", particularmente no que tange aos serviços de atenção primária à saúde. É preciso intervir nos aspectos relacionados à vulnerabilidade programática, de modo a garantir a acessibilidade e, assim, diminuir a iniquidade tão presente nesse segmento populacional (Carneiro Junior e Silveira, 2003; Jesus, 2006).

\section{O Projeto A Gente na Rua}

O Centro de Saúde Escola Barra Funda Dr. Alexandre Vranjac (CSEBFAV) - unidade básica de saúde da Irmandade da Santa Casa de Misericórdia de São Paulo - atende uma população moradora e usuária de distritos administrativos localizados na região central do município de São Paulo. Desde 1995, começou a sistematizar ações voltadas para a atenção à população em situação de rua (Carneiro Junior e col., 2006).

Resultante de várias articulações e projetos setoriais e intersetoriais, como a implantação da Casa de Cuidados Carolina Maria de Jesus (destinada a indivíduos em situação de rua egressos de serviços médico-hospitalares), teve início, a partir de 2003, o Programa de Agentes Comunitários de Saúde, constituindo-se um projeto piloto cuja populaçãoalvo eram os usuários dos serviços sociais da região de abrangência do CSEBFAV e/ou de seu próprio atendimento. Esse projeto, sediado no CSEBFAV, foi estabelecido através de "Termo de Convênio" entre a Secretaria Municipal da Saúde de São Paulo e a Irmandade da Santa Casa de Misericórdia de São Paulo, com a contratação de uma enfermeira e de um agente comunitário de saúde egresso da situação de rua (Silva, 2007; Silva e col., 2007).

Nesse contexto de organização de práticas de saúde específicas, orientado por políticas públicas equânimes, e também em resposta ao "Dia de Luta da População em Situação de Rua", mobilização social ocorrida em junho de 2003 que reivindicava acesso aos serviços sociais, incluindo os da saúde, surge a partir de 2004 o projeto "A Gente na Rua", tendo como estratégia tecnoassistencial a Saúde da Família (São Paulo, 2004).

A implantação desse projeto, inicialmente operacionalizado através do Programa Agente Comunitário de Saúde, pode ser considerada uma experiência singular, transformadora de valores e concepções, exemplificada por uma série de adequações aos processos de trabalhos conhecidos. A começar pelos próprios agentes comunitários (egressos do segmento social em situação de rua), passando pela articulação intersetorial, que se torna uma exigência, até a necessidade de elaboração de novos termos e conceitos. Desse modo, "visita domiciliar" passa a ser "visita de rua"; domicílio, "o lugar em que se costuma ficar na rua”, e família aquela "constituída por indivíduos declarados no momento do cadastro" (cadastro que contemple particularidades como existência de animal de estimação, locais para refeições e higiene, entre outros aspectos).

Tais adequações foram necessárias devido às singularidades do "modo de andar a vida" dos indivíduos em situação de rua, requerendo novas aprendizagens do processo de trabalho e novas maneiras de organizar o serviço.

Em novembro de 2004, o projeto "A Gente na Rua" foi implantado em sete Unidades Básicas de Saúde (UBS), nas regiões das subprefeituras Sé, Mooca e Pinheiros, contando com 11 Agentes Comunitários de Saúde de Rua (ACSR) e uma enfermeira, ampliando, assim, o programa acima mencionado.

Entre outras ações, eram, inicialmente, de responsabilidade do Agente Comunitário de Saúde de Rua (São Paulo, 2004): 
1) acompanhar em média 200 pessoas em seu local de moradia, vinculando-as às Unidades Básicas de Saúde;

2) desenvolver ações básicas de saúde nas áreas de atenção em todas as fases do ciclo de vida e nos projetos prioritários, com ênfase na promoção da saúde e prevenção de doenças, mobilizando a população atendida com vistas à ampliação de autonomia na saúde.

Para isso, a equipe deveria:

1) prestar assistência in loco, quando houvesse necessidade;

2) atuar de forma integrada com os diversos segmentos da comunidade, na perspectiva de estabelecer canais de diálogo, criando vínculos e compromissos compartilhados na tarefa de promover a saúde.

Para a capacitação específica da equipe, foi fundamental a incorporação de temáticas e reflexões sobre a situação de rua, peculiaridades do processo saúde-doença-cuidado nessa população, características dos serviços, possíveis resistências; questões desafiadoras tanto na organização quanto no desenvolvimento do projeto. Além do estabelecimento de um processo de supervisão técnica intensiva e particularizada, pois alguns agentes apresentaram necessárias intervenções assistenciais, em especial no campo da saúde mental.

No ano de 2005, o número de equipes subiu para três, com um total de 35 Agentes Comunitários de Saúde (ACS) nas mesmas regiões. Segundo dados de dezembro de 2007, havia 5.0oo pessoas cadastradas nas regiões em que o projeto estava implantado (Secretaria Municipal da Saúde, 2007). Em 2008, as equipes de PACS foram transformadas em equipes de PSF, perfazendo um total de nove, com 57 ACS, além da contratação de três médicos e seis auxiliares de enfermagem. Dados de dezembro de 2008 já apontam 11.406 pessoas cadastradas, compondo um universo de 7.677 famílias (Secretaria Municipal da Saúde, 2008).

Comparando os dados do Sistema de Informação da Atenção Básica (SIAB) da população em geral do município de São Paulo, cadastrada pelas equipes da Saúde da Família, com os da população em situação de rua, para o período de janeiro de 2007, é possível observar certas diferenças entre esses dois grupos populacionais: as gestações ocorrem seis vezes mais que na população em situação de rua, assim como o alcoolismo incide 30 vezes mais e a tuberculose 57 vezes mais que na população em geral. Embora em termos absolutos a quantidade seja muito menor, proporcionalmente os números revelam o tamanho da vulnerabilidade dos indivíduos em situação de rua (Secretaria Municipal da Saúde, 2007).

\section{Considerações Finais}

Nos últimos anos, implantou-se, na cidade de São Paulo, uma política pública específica para a atenção à população de rua através da Estratégia de Saúde da Família. Iniciativa que tem sido bem-sucedida em favorecer o acesso desse segmento à atenção básica por meio de trabalhos específicos, da atuação em logradouros públicos e/ou albergues e do estabelecimento de vínculos e acompanhamento dos cuidados necessários aos indivíduos em situação de rua.

Outro aspecto a ser ressaltado é o esforço para a superação de ações isoladas, a fim de que elas se pautem na premissa de um trabalho intersecretarial, já que a parceria é fundamental no enfrentamento dos determinantes do processo saúde-doença. Ainda em relação à população em situação de rua, essa parceria torna-se condição intrínseca no sentido de assistir integralmente um grupo tão específico e diversificado.

Contudo, para o aprofundamento de futuros trabalhos na atenção à saúde desse segmento, há ainda que se observar uma série de questões que, embora tenham sido apontadas, não foram aqui plenamente desenvolvidas: o acesso às ações de saúde na perspectiva da integralidade e, neste caso, a efetividade do segmento longitudinal dos indivíduos em situação de rua; a organização do cuidado para os que apresentam transtornos mentais, com abordagens terapêuticas mais específicas, como o gerenciamento de casos ou o estabelecimento de acompanhantes terapêuticos; a nova orientação da Secretaria Municipal da Saúde de que os novos Agentes Comunitários de Saúde não precisam ser egressos desse segmento populacional; e a efetivação da política intersecretarial tanto em nível micro - discussões conjuntas entre os agentes públicos das áreas sociais sobre plano de cuidados, dificuldades 
no manuseio de certas situações apresentadas pelos indivíduos em situação de rua, por exemplo - como em nível macro - criação de centros de serviços e oportunidades de trabalho, moradia, educação, entre outros.

\section{Referências}

ADAY, L. A.; ANDERSEN, R. A framework for the study of access to medical care. Health Services Research, Chicago, v. 9, n. 3, p. 208-20, 1974.

BRASIL. Ministério da Saúde. Saúde da Família. Disponível em: <http:// www.saude.gov.br/ saudedafamilia>. Acesso em: og fev. 2009.

CARNEIRO JUNIOR, N. et al. Serviços de saúde e população de rua: contribuição para um debate. Saúde e Sociedade, São Paulo, v. 7, n. 2, p. 47-62, ago./dez.1998.

CARNEIRO JUNIOR, N.; SILVEIRA, C. Organização das práticas de atenção primária em saúde no contexto dos processos de exclusão/inclusão social. Cadernos de Saúde Pública, Rio de Janeiro, v. 19, n. 6, p. 1827-35, nov./dez. 2003.

CARNEIRO JUNIOR, N. et al. Organização de práticas de saúde equânimes em atenção primária em região metropolitana no contexto dos processos de inclusão e exclusão social. Saúde e Sociedade, São Paulo, v. 15, n. 3, p. 30-39, set./dez. 2006.

CREVELIM, M. A. A Participação da comunidade no Programa Saúde da Família na perspectiva do trabalho em equipe. São Paulo, 2004. Dissertação (Mestrado em Administração de Enfermagem) - Escola de Enfermagem, Universidade de São Paulo, São Paulo, 2004.

FRENK, J. El concepto y la medicion de accesibilidad. In: WHITE, K. L. (Ed.). Investigaciones sobre servicios de salud: una antologia. Washington-D.C: OPS, 1992. p. 929-43.

GIOVANELLA, L.; FLEURY, S. Universalidade da atenção à saúde: acesso como categoria de análise. In: EIBENSCHUTZ, C. (Org.). Política de saúde: o público e o privado. Rio de Janeiro: FIOCRUZ, 1996. p. 177-98.
HORTALE, V. A.; CONILL, E. M.; PEDROZA, M. Desafios na construção de um modelo para análise comparada da organização de serviços de saúde. Cadernos de Saúde Pública, Rio de Janeiro, v. 15, n. 1, p. 79-88, jan./mar. 1999.

HORTALE, V. A.; PEDROZA, M.; ROSA, M. L. G. Operacionalizando as categorias acesso e descentralização na análise de sistemas de saúde. Cadernos de Saúde Pública, Rio de Janeiro, v. 16, n. 1, p. 231-39, jan./mar. 2000.

JESUS, C. H. Estudo descritivo da população adulta em situação de rua da área central do Município de São Paulo relativo à vulnerabilidade às infecções sexualmente transmissíveis e à AIDS. São Paulo, 2006. Dissertação (Mestrado em Saúde Coletiva) - Faculdade de Ciências Médicas da Santa Casa de São Paulo, São Paulo, 2006.

PENCHANSKY, R.; THOMAS, J. W. The concept of access: definition and relationship to consumer satisfaction. Medical Care, Philadelphia, v. 19, n. 2, p. 127-40, feb. 1981.

SÃO PAULO (Município). Prefeitura do Município de São Paulo. Secretaria Municipal da Assistência Social. Fundação e Instituto de Pesquisa. Estimativa do número de pessoas em situação de rua da cidade de São Paulo em 2003. São Paulo: Secretaria Municipal de Assistência Social, 2003. SÃO PAULO (Município). Prefeitura do Município de São Paulo. Secretaria Municipal da Saúde. Secretaria Municipal de Assistência Social. Protocolo de Intenções entre as Secretarias Municipais de Saúde e de Assistência Social para a Atenção à População em Situação de Rua. São Paulo: Secretaria Municipal de Assistência Social, 26 de maio de 2004.

SÃO PAULO (Município). Prefeitura do Município de São Paulo. Secretaria Municipal da Saúde. Sistema de Informação da Atenção Básica. Consolidado das famílias cadastradas e das equipes no ano de 2007. São Paulo: Secretaria Municipal da Saúde, 2007. 
SÃO PAULO (Município). Prefeitura do Município de São Paulo. Secretaria Municipal da Saúde. Sistema de Informação da Atenção Básica. Consolidado das famílias cadastradas e das equipes no ano de 20o8. São Paulo: Secretaria Municipal da Saúde, 2008.

SILVA JUNIOR, A. G. Modelos tecnoassistenciais em saúde: o debate no campo da Saúde Coletiva. São Paulo: Hucitec, 1998.

SILVA, J. B. T. Uma experiência inovadora de política de inclusão social. In: CARACIOLO, J. M. M.; SHIMMA, E. (Orgs.) Adesão da teoria à prática. São Paulo: Secretaria de Estado da Saúde de São Paulo, 2007. p. 260-264.

SILVA, N. L.; SILVA, J. B. T.; JESUS, C. H. O cuidado à saúde da população em situação de rua. In:

CARACIOLO, J. M. M.; SHIMMA, E. (Orgs.) Adesão da teoria à prática. São Paulo: Secretaria de Estado da Saúde de São Paulo, 2007. p. 269-282.
STARFIEL, B. Atenção primária: equilíbrio entre necessidades de saúde, serviços e tecnologia. Trad. Fidelity Translations. Brasília: UNESCO, Ministério de Saúde, 2002.

TRAVASSOS, C. et al. Desigualdades geográficas e sociais na utilização de serviços de saúde no Brasil. Ciência \& Saúde Coletiva, Rio de Janeiro, v. 5, n. 1, p. 135-49, 2000.

VIANA, A. L. D.; DAL PÓZ, M. R. Reforma em saúde no Brasil: programa de saúde da família; informe final. Rio de Janeiro: UERJ, IMS, 1998. (Série Estudos em Saúde Coletiva, n.166).

VIEIRA, M. A.; BEZERRA, E. M.; ROSA, C. M. M. (Orgs.). População de rua: quem é, como vive, como é vista. 3. ed. São Paulo: Hucitec, 2004.

WALLACE, S. P.; ENRIQUEZ-HAASS, V. Disponibilidad, accesibilidad y aceptabilidad en el sistema de atención médica en vías de cambio para los adultos mayores en los Estado Unidos. Reista Panamericana de Salud Publica, Washington, v. 10, n. 1, p. 18-28, jul. 2001. 